\title{
In office pharmacy and its relation to access to care in workers' compensation
}

\section{Introduction}

Workers' Compensation traces its origins back to Germany, where Chancellor Otto Von Bismarck introduced a compulsory state-run accident compensation system in 1884. Nine states passed workers' compensation legislation in 1911, and by 1948 every state had some form of workers' comp law on the books. Workers' Compensation is a state mandated no-fault system form of insurance that ensured that workers injured at work would receive compensation without delay and without regard to fault (820 ILCS 305/1(a)(3)). ${ }^{1}$ It was started in response to serious societal problems caused by a dramatic rise in the number of people injured in industrial settings. It is a benefit provided in exchange for mandatory relinquishment of the employee's right to sue his or her employer under the common civil law of negligence. ${ }^{2}$

The relationship between the legitimately injured/sickened worker and the insurance carrier paying the medical bills and other compensation is by nature somewhat adversarial. The injured worker wants quality medical treatment to enable a full recovery, and the insurance carrier wants as inexpensive resolution as possible. Disputes can arise when the claims administrator contests employee claims. We have found that, in certain social climates, barriers to care prevent the appropriate implementation of the spirit and legislative intent of the Workers Compensation system.

There are many issues that create an environment that limits timely access to appropriate treatment for the injured worker. We have previously reviewed our workers compensation accounts receivables in our orthopedic practice from 2006 until $2014 .{ }^{3}$ The collection rate for the workers compensation population was $68 \%$ (32\% of claims never paid) while the average time in collections was 3.6 years ( 1,342 days) for active claims. Review of the statistical data demonstrated that $98 \%$ of all accounts receivable (AR) belonged to workers compensation $(87 \%)$ patients and personal injury claims $(11 \%)$, even though they represented $58.8 \%$ and $5.2 \%$ of the total claims respectively. Only $2 \%$ of AR was the result of PPO, BCBS or Medicare. When providers provide a service, they incur a cost. If there is a chance that they may not be compensated for a service provided, they must make a decision to provide or withhold the service. This inherently creates a barrier to care for the injured worker that does not exist for other patients.

We conducted a consecutive case series to analyze access to appropriate medications for workers' compensation patients. Most treatment guidelines require an initial attempt at conservative management prior to advancing treatment. This often includes the use of non-steroidal anti-inflammatories (NSAIDS) and other medications. Many physician offices have added pharmacies to their service lines to both act as a profit center and provide a valuable service to their patients. Many treaters see the pharmacy as an extension of their care not unlike physical therapy, x-ray, MRI, durable medical equipment (DME) and surgery centers. When barriers to access to treatment are erected by insurers, it is not uncommon for the patient's only access to appropriate medications to be from the doctor's office or paying out of pocket.

\author{
Volume 10 Issue 3 - 2018 \\ Blair A Rhode and William S Rhode \\ Orland Park Orthopedics, USA
}

Correspondence: Blair A Rhode, Orland Park Orthopedics, 16450 S 104th AV, Orland Park, IL 60467, Fax 708-364-8443, Tel 708-364-844I,Email Blairbones@gmail.com

Received: April 16, 2018| Published: May 01, 2018

\section{Methods}

We analyzed a consecutive series of worker's compensation patients within our clinic to evaluate their access to appropriate medications. All of the patient's conditions had the use of an oral NSAID listed as a first line therapy by the Official Disability Guides (ODG). Six consecutive patients were given a prescription for an NSAID and $\mathrm{H} 2$ blocker and told to fill them at the local pharmacy. Their patient face sheet was printed to assist the pharmacy with the appropriate information. We collected data on access to appropriate medications. We recorded if the prescription was filled, when it was filled and medication costs. We compared the consecutive case series to patients that received their medication from our pharmacy.

\section{Results}

Of the six patients, only one patient had their prescription fully filled by the pharmacy. A second patient had one of the two medications filled. Both of the patients that received prescriptions did not receive them the day they first presented to the pharmacy. One had to return two days later and another had to return 4 days later after the pharmacy received authorization. The other patients were refused the medication without paying for them out of pocket. The average charge for the NSAID was $\$ 59.99$ while the H2 blocker was $\$ 306.99$. Further complicating the process was the fact that three of the six patients had what they described as serious transportation issues. All patients that received their medication from our office experienced no refusals, no delays, and no personal cost to obtain their medication.

\section{Discussion}

The primary stakeholder in workers compensation is the injured worker seeking timely access to care and the employer/insurer tasked with providing this access. Although cost of delivery of care should always be a consideration, the spirit of our no-fault system is to provide timely access to appropriate care. We have demonstrated that the workers compensation system is one of a severe power imbalance of the insurer and the injured worker. The injured worker is often financially neutral prior to their injury. Once they are injured, they are often placed in a financially desperate position that is further complicated by access to medical care issues. We believe that physicians that are able to bundle service lines including an in-house pharmacy provide an invaluable service to the injured worker. 
It is important to realize that the treating physician has an ethical responsibility to respect their patient's health and interests. In a system that often places barriers to access through delay and denial maneuvers, the physician is often the only advocate the patient has. Timely access has been shown to be good business as well. A prior study analyzing early access to treatment for manual laborers with compressive neuropathies was shown to decrease medical costs in surgical patients by as much as $\$ 250,000 .{ }^{4}$ Patients that experienced a delay in treatment due to a denial process took on average 47 months to reach maximal medical improvement (MMI) compared to the patient group that received appropriate, timely treatment achieved MMI within 5.7 months.

This study demonstrates that difficulty with access to appropriate conservative treatment methods is the norm when the injured worker is told to fill a prescription at a local pharmacy. The pharmacy protocol is to typically authorize the medication with the insurance company that represents the employer of the injured worker. As demonstrated by this study, every injured worker experienced a denial and delay in access to care when their prescriptions were not filled at the doctor's office. This often results in a snowball effect to further denial of treatment when the physician determines the need for a more invasive treatment. The utilization review (UR) process often denies second line treatment when ODG guidelines for conservative treatment are not met.

Of interest, there are medical groups that that advertise that they do not offer an in-house pharmacy as a marketing tool to insurers (https://www.ibji.com/services/workers-comp-related-services/). They suggest that there is a conflict when the physician's office fills prescriptions in the office. It is interesting that the same physician's group advertizes that they have their own physical therapy, radiology, DME and surgery centers. All of these profit centers that are provided by this group has the potential to be over utilized and abused. A physician recommending surgery has the potential for overutilization. Spinal fusion rates in the United States are about double the rate in Canada, Western Europe, and Australia, and about 5 times the rate in the United Kingdom. ${ }^{5}$ Ultimately, we have to trust that our physicians are ethical and moral people that recommend treatments based upon what is most appropriate for the patient. The social compact that the injured worker agreed to when they relinquished their right to sue in civil court was to receive appropriate treatment without delay and in a no fault environment. In an environment where the power imbalance tilts away from the injured worker, a system that gives timely access to unimpeded healthcare is not only essential, it is good business.

\section{References}

1. (820 ILCS 305/) Workers' Compensation Act.

2. Bible JE, Spengler DM, Mir JM. A primer for workers' compensation. Spine J. 2014;14(7):1325-1331.

3. Rhode BA. The Three D's of Insurance: Delay, Deny and Do Not Pay Analysis of Collection Rates for Illinois Workers' Compensation Patients. MOJ Orthop Rheumatol. 2015;3(2):00085.

4. Blair AR, William SR, Brent R. Removal of Barriers to Care Result in Improved Outcomes for Manual Laborers with Compressive Neuropathies. MOJ Orthop Rheumatol. 2015;2(2):00041.

5. Lee J. Rethinking Spine Care. Modern Healthcare. 2014. 